\title{
OPEN CORES FOR DIGITAL SIGNAL PROCESSING
}

\author{
NÚCLEOS LIBRES PARA PROCESAMIENTO DIGITAL DE SEÑALES
}

Juan Camilo Valderrama-Cuervo', Alexander López-Parrado².

1. Universidad del Quindío - Colombia.

2. Universidad del Quindío, Universidad del Valle - Colombia.

Recibido: 07 de febrero de 2014

Aceptado: 27 de marzo de 2014

*Correspondencia del autor. Carrera 15 Calle 12 Norte, Universidad del Quindío, Código Postal 630004, Bloque de Ingeniería, Tercer Piso, CEIFI, Armenia, Colombia. Tel: 3108282506.

Email:parrado@uniquindio.edu.co, alexander.lopez.parrado@correunivalle.edu.co.

\begin{abstract}
This paper presents the design and implementation of three System-on-Chip (SoC) cores, which implement the Digital Signal Processing (DSP) functions: Finite Impulse Response (FIR) filter, Infinite Impulse Response (IIR) filter and Fast Fourier Transform (FFT). The FIR-filter core is based on the symmetrical realization form, the IIRfilter core is based on the Second Order Sections (SOS) architecture and the FFT core is based on the Radix $2^{2}$ Single Delay Feedback (R2 ${ }^{2} \mathrm{SDF}$ ) architecture. The three cores are compatible with the Wishbone SoC bus, and they were described using generic and structural VHDL. In-system hardware verification was performed by using an OpenRisc-based SoC synthesized on an Altera FPGA. Tests showed that the designed DSP cores are suitable for building SoC based on the OpenRisc processor and the Wishbone bus.
\end{abstract}

Keywords: Digital signal processing, digital filters, finite impulse response filters, infinite

\section{RESUMEN}

Este artículo presenta el diseño e implementación de tres núcleos para sistemas en un solo chip (SoC) que implementan las funciones de procesamiento digital de señales (DSP): filtro de respuesta finita al impulso (FIR), filtro de respuesta infinita al impulso (IIR) y transformada rápida de Fourier (FFT). El núcleo de filtro FIR está basado en la estructura simétrica, el núcleo de filtro IIR está basado en la arquitectura de secciones de segundo orden (SOS) y el núcleo de la FFT está basado en la arquitectura base $2^{2}$ Single Delay Feedback (R2 $\left.{ }^{2} \mathrm{SDF}\right)$. Los tres núcleos son compatibles con el bus para SoC Wishbone y fueron descritos usando VHDL estructural y genérico. Se realizó una verificación en hardware usando un SoC basado en el procesador OpenRISC y sintetizado en un FPGA de Altera, las pruebas mostraron que los núcleos DSP son apropiados para construir un SoC basado en el procesador OpenRISC y el bus Wishbone.

Palabras clave: Procesamiento digital de señales, filtros digitales, filtros de respuesta al impulso finita, filtros de respuesta al impulso infinita, transformada rápida de Fourier, sistemas en un solo chip, hardware de código abierto, procesador OpenRISC, bus Wishbone. 


\section{INTRODUCTION}

Today's technology uses heavily Digital Signal Processing (DSP) on its applications, and since the past 20 years (1) these applications have been growing up because the performed improvements to digital integrated circuits in speed, integration capabilities and power consumption. The increased speed of integrated circuits allows real time processing of signals with higher bandwidths such as the ones used in communication systems (1).

Nowadays there are Digital Signal Processors (DSPs) devices specifically designed for DSP that perform real time filtering, Fourier transforms, Wavelet transforms, or encoding processes on audio and video signals. Nevertheless, the parallel nature of DSP algorithms has motivated research interest to hardware solutions based on reconfigurable targets such as the Field Programmable Gate Arrays (FPGAs); these solutions have demonstrated improvements in speed and power consumption compared with the DSPsbased ones (2).

There are several FPGA-based DSP solutions, which are developed by private corporations such as Altera and Xilinx. These solutions include FIR filtering cores $(3,4)$, FFT cores $(5,6)$, among others; however these cores have expensive licenses for commercial use or they can be used for free only for academic purposes.

Nonetheless, a new open source hardware development model inspired from open source software models has been deployed since the last ten years. This model has been supported by communities like OpenCores, which develops open source hardware under the Lesser General Public License (LGPL). OpenCores community has remarkable products as the OpenRISC processor core (7) and the Wishbone bus specification (8), which jointly allow the development of SoC hardware. However, OpenCores community lacks of fully parameterizable DSP cores compatible with the Wishbone bus.

By considering previous ideas, we developed cores FIR filter, IIR filter and FFT under the LGPL license, which are compatible with the Wishbone bus and allow the development of DSP-SoC based on the OpenRISC processor (9). The FIR-filter core is based on the symmetrical architecture $(1,2)$, the IIR core is based on the SOS architecture $(1,2)$, and the FFT core is based on the R2 ${ }^{2} \mathrm{SDF}$ architecture (10). The three cores were described using generic and structural VHDL and targeted to an Altera FPGA device.

This paper is organized as follows: First, some theoretical concepts about DSP and Wishbone bus are described, then the design of the DSP cores architecture is presented and its functional blocks are described, later the in-system hardware verification results are discussed, and finally the conclusion and the acknowledgements are presented.

\section{THEORETICAL BACKGROUND}

This section presents some theoretical concepts about the DSP functions that were implemented, and the SoC bus Wishbone.

\section{FIR Filters}

FIR filters are discrete Linear Time Invariant (LTI) systems that have a finite duration impulse response $h[n]$. When $h[n]$ is symmetrical the FIR filter has linear phase (1) leading to a constant group delay. Practical implementations of FIR filters are always stable because of their non-recursive nature. Eq. (1) shows the direct realization of a FIR filter.

$$
y[n]=\sum_{k=0}^{N-1} h[k] x[n-k]
$$

Here, $x[n]$ is the input signal, $y[n]$ is the output signal and $\mathrm{N}$ is the length of the impulse response $\mathrm{h}[\mathrm{n}]$. There are several realization forms for FIR filters [1]; the direct form, the symmetrical form, and the transpose form are the most used [1]. In the case of hardware implementations, the transpose form has the shortest critical path and it is less sensitive to the round-off errors when fixed point arithmetic is used [1][2]. Figure 1 shows the transpose realization form for a FIR filter with impulse response of length N. From Figure 1 it can be seen that the critical path of the transpose realization form is determined by the combinatorial elements multiplier and adder. The transpose realization form uses N-1 registers.

\section{IIR Filters}

IIR filters are discrete Linear Time Invariant (LTI) systems that have an infinite duration impulse response. Practical implementations of IIR filters can become unstable because of their recursive nature [1]. Eq. (2) shows the direct realization of a (M-1)-th order IIR filter. 


$$
y[n]=\sum_{k=0}^{M-1} b_{k} x[n-k]-\sum_{k=1}^{M-1} a_{k} x[n-k]
$$

Here, $b_{k}$ is the coefficients set for the non-recursive part, $a_{k}$ is the coefficients set for the recursive part, $x[n]$ is the input signal, and $y[n]$ is the output signal. There are several realization forms for IIR filters [1]; the direct form, the type II form, the transpose type II form, and the SOS form are the most used [1]. The transpose type II form has the shortest critical path; nonetheless the SOS form is less sensitive to the round-off errors when fixed point arithmetic is used [1] [2]. In the case of hardware implementations the SOS form has good stability for high order filters, and the critical path is minimized by using the transpose type II form for each second-order section. Figure 2 shows the transpose type II realization form for a single second-order section. Here, $\mathrm{N}_{\text {sect }}$ is the number of second-order sections and $\mathrm{G}$ is the total gain after the SOS decomposition [1], thus each second-order section has a gain of ${ }^{\text {Nsect }} \sqrt{G}$.

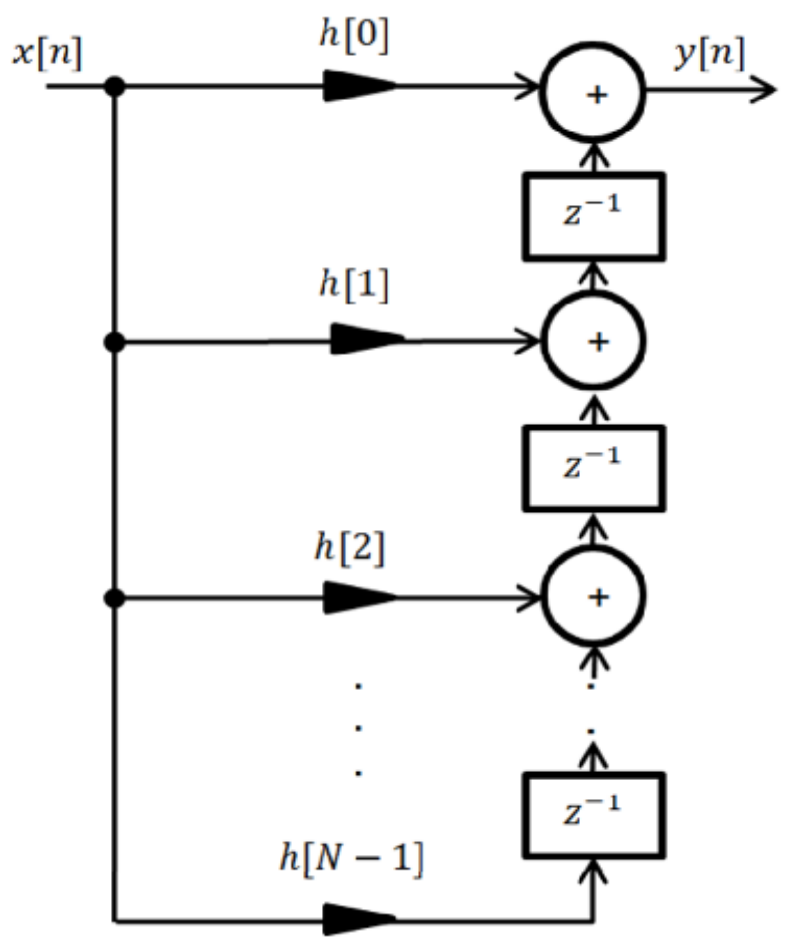

Figure 1: Transpose realization form for a FIR filter of length $\mathbf{N}$.

The whole IIR filter is composed by a cascade of $\mathrm{N}_{\text {sect }}$ second-order sections as the shown in Figure 2. From Figure 2 it can be seen that the critical path of the transpose type II realization form is determined by two multipliers and two adders.

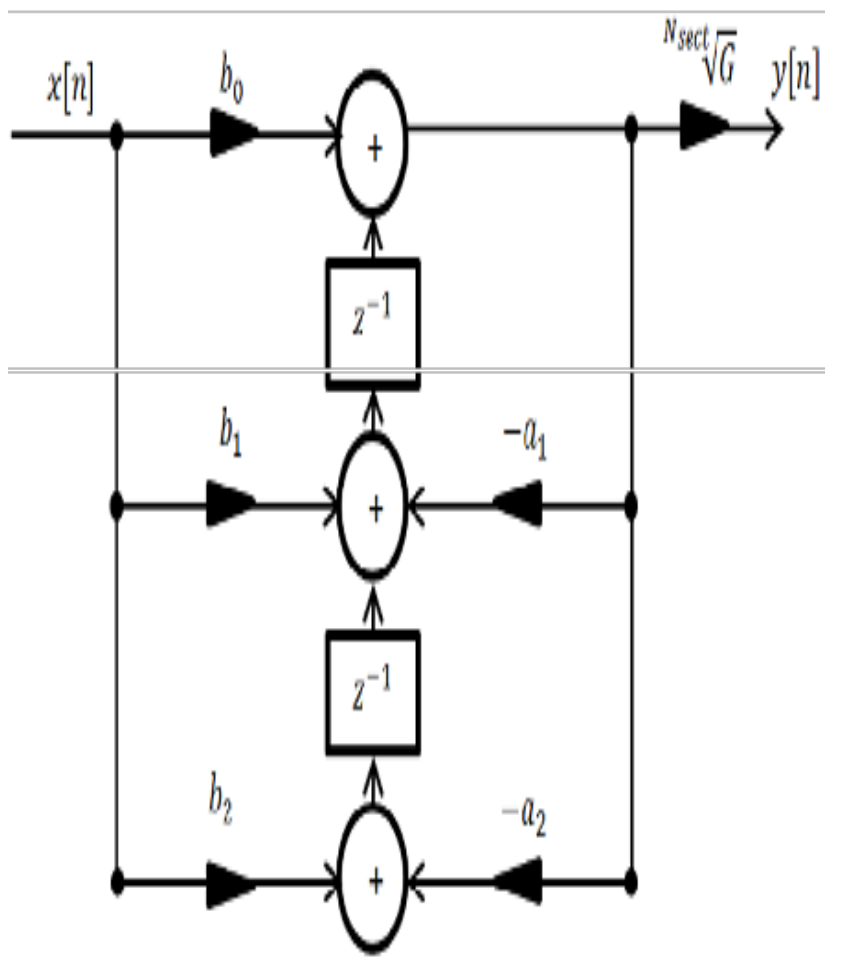

Figure 2: Transpose type II realization form for second order section.

The whole IIR filter is composed by a cascade of $\mathrm{N}$ sect second-order sections as the shown in Figure 2. From Figure 2 it can be seen that the critical path of the transpose type II realization form is determined by two multipliers and two adders.

\section{Fast Fourier Transform}

The FFT is an algorithm that efficiently computes the Discrete Fourier Transform (DFT) of a discrete time signal [1] [2]. The DFT of a signal $x[n]$ is shown in Eq. (3)

$$
X[k]=\sum_{n=0}^{N-1} x[n] e^{-\frac{i 2 \pi k n}{N}}
$$

According to the used radix, FFT algorithms can be radix-2, radix-4, radix- $2^{2}$, radix-8, mixed-radix, split-radix $[2][5][6][10]$, among others. The radix-22 algorithms have become popular for hardware implementations of the FFT [5][6][10] due to their regularity, simple control, pipelined operation, and low hardware resources usage; the R2 ${ }^{2} \mathrm{SDF}$ architecture is based on a radix-22 algorithm and it is suitable for FFT hardware [5][6][10][11]. Figure 3 shows the $\mathrm{R} 2{ }^{2} \mathrm{SDF}$ architecture for a 64-point FFT. The R2 ${ }^{2} \mathrm{SDF}$ architecture uses two types of butterflies, which have a similar structure to the radix-2 butterfly, the R2 ${ }^{2} \mathrm{SDF}$ architec- 
ture has a resource usage similar to the radix- 4 algorithms [10]. From Figure 3 it can be seen that control is performed by a $\log _{2}(n)$-bit counter.

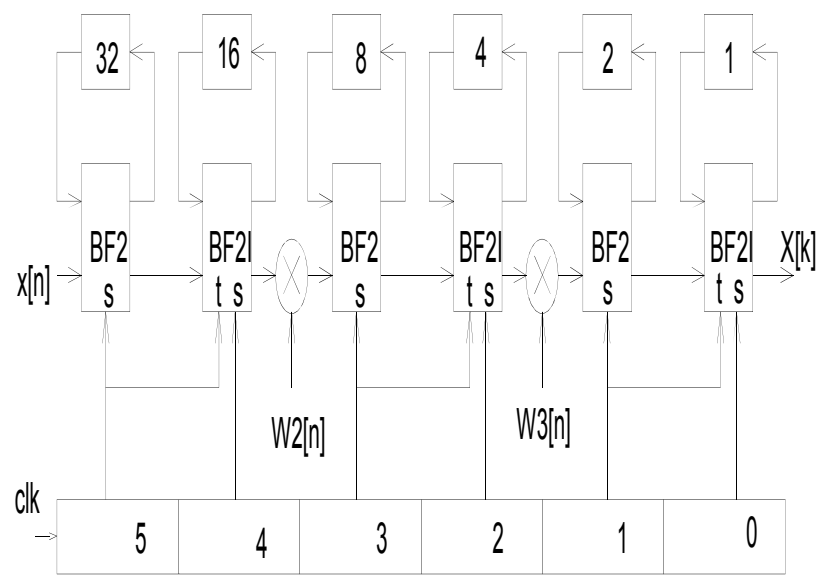

Figure 3: R22SDF architecture for 64-point FFT.

\section{OpenRISC processor and the Wishbone bus}

OpenRISC is a Reduced Instruction Set Computer (RISC) 32-bit soft-core processor designed by OpenCores community, its architecture is described in a standard document [7]; also a synthesizable description in Verilog under the LGPL license is available through the OR1200 core [12]. OpenRISC allows the development of SOCs by using the interconnection bus Wishbone which is described in a standard document [8]. The OpenCores community has developed numerous cores with Wishbone connectivity such as Universal Asychronous Receiver Transmitter (UART), memory controller, Ethernet controller, timer controller, among others; however the OpenCores community has no DSP cores with Wishbone connectivity. Figure 4 shows a basic Wishbone interconnection between a master device and a slave device. Here, the master is either the OpenRISC processor or a bus controller; the slave is any Input/Output (I/O) device, coprocessor or hardware accelerator. According to the Wisbone specification [8] the signals in Figure 4 are described in Table 1 . The DSP cores proposed in this papers are Wishbone compatible, and they use the basic connection depicted in Figure 4. The OpenCores community has developed some reference SoCs based on the OpenRISC processor which are FPGA-synthesizable; one of the simplest is MinSoC [13], which allows an easy and fast verification of the OpenRISC-based SoC with custom slave modules such as the DSP cores we designed.

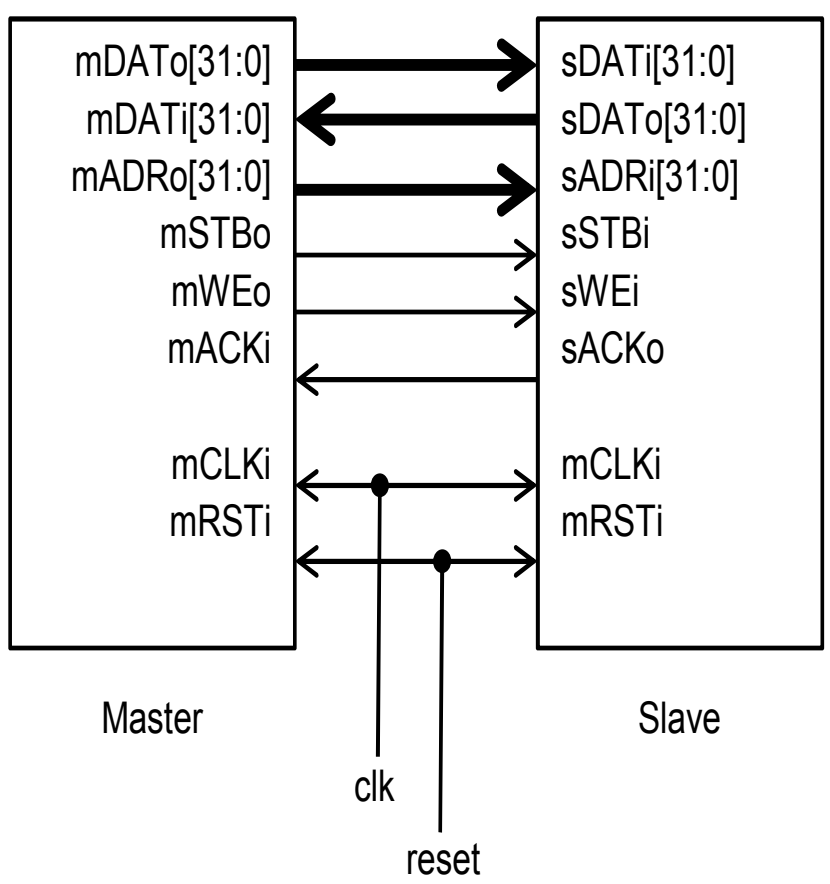

Figure 4: Wishbone bus basic connection.

\begin{tabular}{|l|l|}
\hline \multicolumn{1}{|c|}{ Signal } & \multicolumn{1}{c|}{ Description } \\
\hline mDATo/sDATi & $\begin{array}{l}\text { The data bus from master to } \\
\text { slave (write operations). }\end{array}$ \\
\hline mDATi/sDATo & $\begin{array}{l}\text { The data bus from slave to } \\
\text { master (read operations). }\end{array}$ \\
\hline mADRo/sADRi & $\begin{array}{l}\text { The address bus from master } \\
\text { to slave. }\end{array}$ \\
\hline mSTBo/sSTBi & $\begin{array}{l}\text { The chip select signal from } \\
\text { master to slave. It is set by } \\
\text { master during a write/read } \\
\text { operation. }\end{array}$ \\
\hline mWEo/sWEi & $\begin{array}{l}\text { The write enable signal from } \\
\text { master to slave. It is set by } \\
\text { master during a write opera- } \\
\text { tion. }\end{array}$ \\
\hline mACKi/sACKo & $\begin{array}{l}\text { The acknowledgment signal } \\
\text { from slave to master. It is set } \\
\text { by slave after a successful } \\
\text { write/read operation. }\end{array}$ \\
\hline clk & The global clock signal. \\
\hline reset & $\begin{array}{l}\text { The global active high reset } \\
\text { signal. }\end{array}$ \\
\hline
\end{tabular}

Table 1: Signal description of Wishbone bus. 


\section{DSP CORES ARCHITECTURE}

In this section we describe the designed DSP cores and the slave interfaces with the Wishbone bus. For all DSP cores we used fixed point arithmetic. Here the word length, guard bit, fractional part [2], filter order, and FFT length [1] are parameterizable features through VHDL generics. Each DSP core is composed of two functional unit, the processing unit and the slave interface unit; the processing unit performs the DSP operation according to the considered core and the slave interface unit is the Wishbone interface for the SoC connection.

\section{FIR-filter core}

Figure 5 shows the block diagram of a FIR-filter core parameterized with $\mathrm{N}=50$, word length of $\mathrm{M}=16$ bits, and a guard bit of $\mathrm{G}=8$ bits. In this case, the processing unit was designed by using the transpose realization

\section{DSP CORES ARCHITECTURE}

In this section we describe the designed DSP cores and the slave interfaces with the Wishbone bus. For all DSP cores we used fixed point arithmetic. Here the word length, guard bit, fractional part [2], filter order, and FFT length [1] are parameterizable features through VHDL generics. Each DSP core is composed of two functional unit, the processing unit and the slave interface unit; the processing unit performs the DSP operation according to the considered core and the slave interface unit is the Wishbone interface for the SoC connection.

\section{FIR-filter core}

Figure 5 shows the block diagram of a FIR-filter core parameterized with $\mathrm{N}=50$, word length of $\mathrm{M}=16$ bits, and a guard bit of $\mathrm{G}=8$ bits. In this case, the processing unit was designed by using the transpose realization form shown in Figure 1. In Figure 5 the unconnected signal ports are Wishbone compatible signals for OpenRISC-based SoC integration. The remaining signals are described as follows: The input_signal/ sdat_o pair is the port with the signal to be filtered and it has a length of $\mathrm{M}+\mathrm{G}$ bits; the output_signal/ sdat_i pair is the port with the filtered signal and it has a length of $\mathrm{M}+\mathrm{G}$ bits; the enable/start pair is the port that enables the filtering process in the processing unit; the filter_coef/HQ pair is the port with the filter coefficients and it has a length of NxM bits; the $\mathrm{Q} / \mathrm{Q}$ pair is the port with the number of fractional bits in the fixed-point representation of the filter coefficients and it has a length of 4 bits. Table 2 shows the register description of the Wishbone interface for the FIR-filter core.

\begin{tabular}{|l|l|}
\hline \multicolumn{1}{|c|}{ Register } & \multicolumn{1}{c|}{ Address } \\
\hline FIR_CONTROL[0:0] & FIR_BASE +0 \\
\hline FIR_DATA[M+G-1:0] & FIR_BASE +4 \\
\hline FIR_STATUS[0:0] & FIR_BASE +8 \\
\hline FIR_Q[3:0] & FIR_BASE +12 \\
\hline FIR_COEFF & FIR_BASE +16 \\
\hline
\end{tabular}

Table 2: Register description of the FIR-filter core.

FIR_DATA is a read/write register used to write/ read the input/filtered sample. FIR_CONTROL is a write-only register; when it is set by the user, the filtering process is started. FIR_STATUS is a readonly register; it is set when the filtering process finishes. FIR_Q is a write-only register; in this address the user writes the number of fractional bits of the fixed-point representation of the filter coefficients. From FIR_COEFF starts an addressing space composed of $\mathrm{N}$ consecutive 32-bit address positions where the user can write the 16-bit fixed-point filter coefficients starting from FIR_COEFF for $h[0]$ and finishing with FIR_COEFF+4 $\mathrm{x}(\mathrm{N}-1)$ for $\mathrm{h}[\mathrm{N}-1]$.

\section{IIR-filter core}

Figure 6 shows the block diagram of a IIR-filter core parameterized with $\mathrm{N}_{\text {sect }}=6$, word length of $\mathrm{M}=16$ bits, a guard bit of $\mathrm{G}=8$ bits, and $\mathrm{Q}=13$ fractional bits. In this case the processing unit was designed by using a cascade of $\mathrm{N}_{\text {sect }}$ pipelined SOS as the shown in Figure 2. In Figure 6 the unconnected signal ports are Wishbone compatible signals for OpenRISC-based SoC integration. The remaining signals are described as follows: The input_signal/sdat_o pair is the port with the signal to be filtered and it has a length of $\mathrm{M}+\mathrm{G}$ bits; the output_signal/sdat_i pair is the port with the filtered signal and it has a length of $\mathrm{M}+\mathrm{G}$ bits; the enable/ start pair is the port that enables the filtering process in the processing unit; the enable_out/enable_in pair is the flag that signals the filtering process completion; the filter_coef/HQ pair is the port with the filter coefficients and it has a length of 6xNsectxM bits; the gain/gain pair is the port with the gain ${ }^{\mathrm{Nsect}} \sqrt{G}$ for each 
SOS and it has a length of M bits; the en_out/en_out pair is the port with the number of used sections minus one from the N_sect available sections, and it has a width of 4 bits. In this case, Q fractional bits are used for the fixed-point representation of the filter coefficients and the gain. Table 3 shows the register description of the Wishbone interface for the IIR-filter core. IIR_DATA is a read/write register used to write/read the input/filtered sample. IIR_CONTROL is a write-only register; when it is set by the user, the filtering process is started. IIR_STATUS is a write/read register; it is set when the filtering process finishes, and it is cleared with a write operation. IIR_NSECT is a write-only register; in this address, the user writes the number of used sections minus one from the Nsect available sections; IIR_GAIN is a write-only register; in this address, the user writes the gain ${ }^{\text {Nsect }} \sqrt{G}$ for each SOS by using fixed-point representation with $Q$ fractional bits. From IIR_COEFF starts an addressing space composed of $6 \mathrm{xN}_{\text {sect }}$ consecutive 32-bit address positions where the user can write the 16-bit fixed-point SOS coefficients starting from IIR COEFF for $\mathrm{a}_{2}-1^{\text {st }}$ SOS section, IIR_COEFF +4 for $\mathrm{a}_{1}-1^{\text {st }}$ SOS section, IIR_COEFF +8 for $\mathrm{a}_{0}-1^{\text {st }}$ SOS section, IIR_COEFF +12 for $b_{2}-1^{\text {st }}$ SOS section, IIR_COEFF $+1 \overline{6}$ for $b_{1}-1^{\text {st }}$ SOS section, and IIR_COEFF +20 for $\mathrm{b}_{0}-1^{\text {st }}$ SOS section; and finishing with IIR_COEFF + $4 \mathrm{x}\left(6 \mathrm{xN}_{\text {sect }}-6\right)$ for a2-N $\mathrm{N}_{\text {sect }}$ st SOS section, IIR_COEFF $+4 \mathrm{x}\left(6 \mathrm{xN}_{\text {sect }}-5\right)$ for a1- $\mathrm{N}_{\text {sect }}$ st SOS section, IIR_CO$\mathrm{EFF}+4 \mathrm{x}(6 \mathrm{xNsect}-4)$ for a0-Nsectst SOS section,

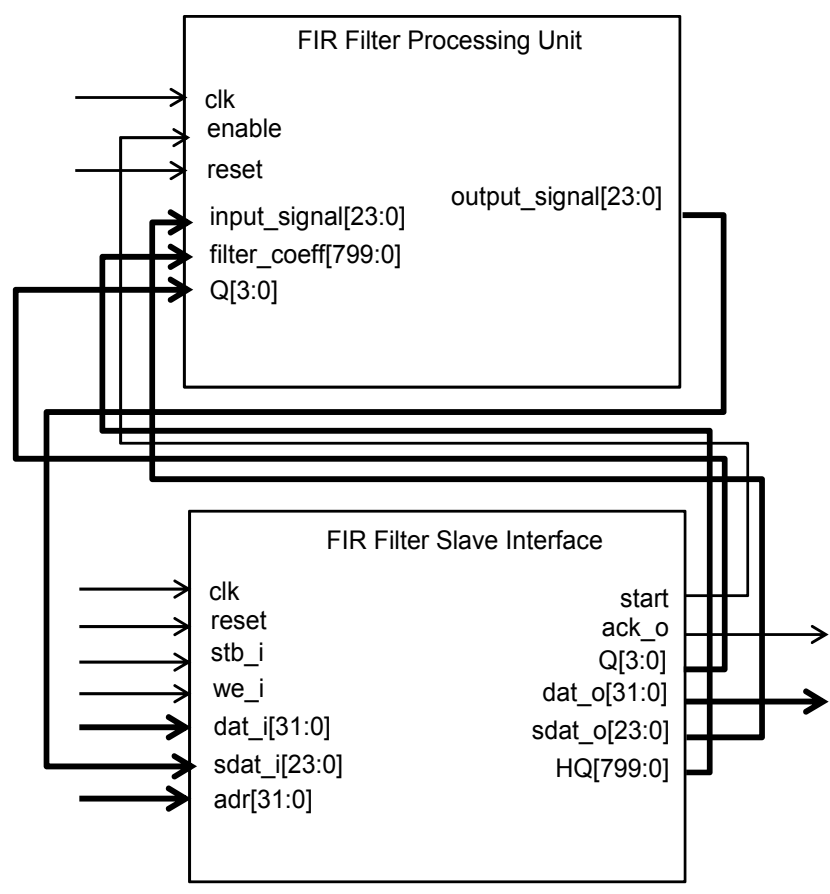

Figure 5: FIR-filter core.
IIR_COEFF $+4 \mathrm{x}(6 \mathrm{xNsect}-3)$ for b2-Nsectst SOS section, IIR_COEFF $+4 \mathrm{x}(6 \mathrm{x}$ Nsect- 2$)$ for b1-Nsectst SOS section, and IIR_COEFF $+4 \mathrm{x}(6 \mathrm{xNsect}-1)$ for b0-Nsectst SOS section.

\begin{tabular}{|l|l|}
\hline \multicolumn{1}{|c|}{ Register } & \multicolumn{1}{c|}{ Address } \\
\hline IIR_CONTROL[0:0] & IIR_BASE + 0 \\
\hline IIR_DATA[M+G:0] & IIR_BASE + 4 \\
\hline IIR_STATUS[0:0] & IIR_BASE + 8 \\
\hline IIR_NSECT[3:0] & IIR_BASE + 12 \\
\hline IIR_GAIN[15:0] & IIR_BASE + 16 \\
\hline IIR_COEFF & IIR_BASE + 20 \\
\hline
\end{tabular}

Table 3: Register description of the IIR-filter core.

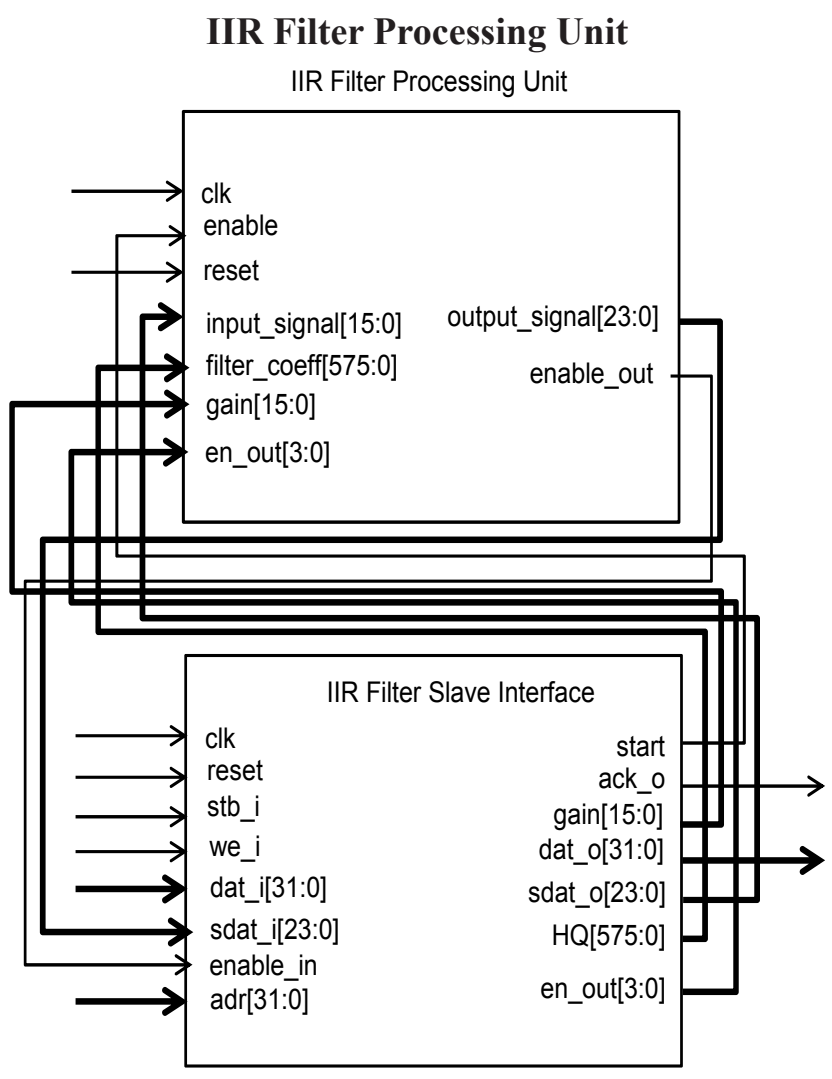

Figure 6: IIR-filter core.

\section{FFT core}

Figure 7 shows the block diagram of an FFT core parameterized with $\mathrm{N}=1024$, word length of $\mathrm{M}=16$ bits, and $\mathrm{Q}=15$ fractional bits. In this case, the processing unit was designed by using the pipelined version of the R22SDF architecture developed in [11]. In Figure 7 , the unconnected signal ports are Wishbone compatible signals for OpenRISC-based SoC integration. 
The remaining signals are described as follows: The Xinr/dat[15:0] pair is the port with the real part of the input sample and it has a length of M bits; Xini/ dat [31:16] pair is the port with the imaginary part of the input sample and it has a length of $\mathrm{M}$ bits; the enable/fft_enable pair is the port that enables the FFT computing for each input sample in the processing unit; the Xoutr/sdat_i[15:0] pair is the port with the real part of the output sample and it has a length of M bits; Xouti/sdat_i[31:16] pair is the port with the imaginary part of the output sample and it has a length of $\mathrm{M}$ bits; the enable_out/fft enable in pair is the flag that signals the FFT process completion for each input sample; the frame_ready/fft_finish pair is the flag that signals the whole FFT process completion; the index/ adr_fft pair

\section{FFT core}

Figure 7 shows the block diagram of an FFT core parameterized with $\mathrm{N}=1024$, word length of $\mathrm{M}=16$ bits, and $\mathrm{Q}=15$ fractional bits. In this case, the processing unit was designed by using the pipelined version of the R22SDF architecture developed in [11]. In Figure 7 , the unconnected signal ports are Wishbone compatible signals for OpenRISC-based SoC integration. The remaining signals are described as follows: The Xinr/dat[15:0] pair is the port with the real part of the input sample and it has a length of $\mathrm{M}$ bits; Xini/ dat [31:16] pair is the port with the imaginary part of the input sample and it has a length of $\mathrm{M}$ bits; the enable/fft_enable pair is the port that enables the FFT computing for each input sample in the processing unit; the Xoutr/sdat_i[15:0] pair is the port with the real part of the output sample and it has a length of M bits; Xouti/sdat i[31:16] pair is the port with the imaginary part of the output sample and it has a length of $\mathrm{M}$ bits; the enable_out/fft enable in pair is the flag that signals the FFT process completion for each input sample; the frame_ready/fft_finish pair is the flag that signals the whole FFT process completion; the index/adr_fft pair is the bit-reversed address [1] [2] in On-chip RAM where the processed sample is written. Table 4 shows the register description of the Wishbone interface for the FFT core. FFT_DATA is a write-only register used to write the input samples. FFT_CONTROL is a write-only register; when it is written by the user the processing unit and the status register are cleared; FFT_STATUS is a read-only register; it is set when the whole FFT process finis- hes. From FFT_MEMORY starts an addressing space composed of $\mathrm{N}$ consecutive 32-bit address positions where the user can read the FFT results starting from FFT_MEMORY for X[0] and finishing with FFT MEMORY $+4 x(N-1)$ for $X[N-1]$.

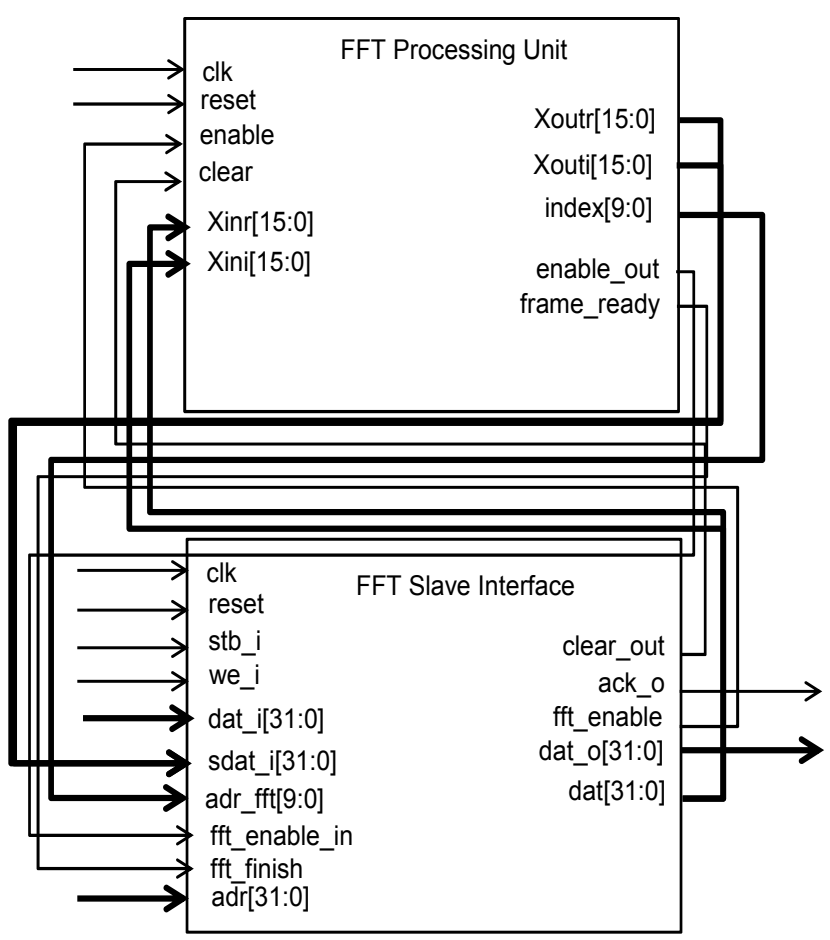

Figure 7: IIR-filter core.

\begin{tabular}{|l|l|}
\hline \multicolumn{1}{|c|}{ Register } & \multicolumn{1}{c|}{ Address } \\
\hline FFT_CONTROL[0:0] & FFT_BASE +0 \\
\hline FFT_DATA[31:0] & FFT_BASE +4 \\
\hline FFT_STATUS[0:0] & FFT_BASE +8 \\
\hline FFT_MEMORY & FFT_BASE +12 \\
\hline
\end{tabular}

Table 4: Register description of the FFT core. 


\section{IN-SYSTEM HARDWARE VERIFICATION}

The three DSP cores were integrated into an OpenRISC-based SoC built from the reference design MinSoC [13]. The SoC was synthesized on the Altera FPGA device EP2S60F1020C4 included in the development board TREX-S2-TMB [14]. The accuracy of the cores was measured in terms of the Mean Squared Error (MSE) between frequency responses in DFT domain as shown in Eq. (4)

$$
M S E=\sum_{k=0}^{N-1}|X[k]-\tilde{X}[k]|^{2}
$$

Here $X[\mathrm{k}]$ is the frequency response in the DFT domain when it is computed by simulation using double precision floating point arithmetic, and $\mathrm{X}^{\tilde{}}[\mathrm{k}]$ is the frequency response of the core in DFT domain.

In the case of the FIR-filter core, we designed a 49-th order equiripple low-pass filter with cutoff frequencies $3 / 8 \pi \mathrm{rad} / \mathrm{s}$ and $\pi / 2 \mathrm{rad} / \mathrm{s}$. The core is parameterized with a 16-bit word length, a 15-bit fractional part, and a guard bit of 8 bits. Figure 8 shows the magnitude frequency responses for the tested FIR-filter core.

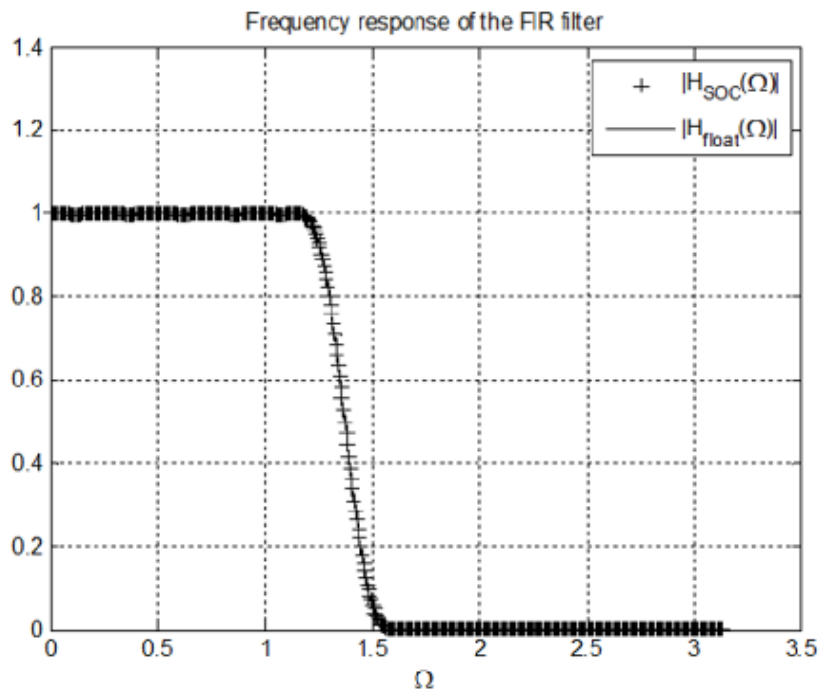

Figure 8: Frequency response of the FIR-filter core.

In Figure 8, the continuous line depicts the magnitude frequency response of the FIR filter when it is computed by simulation using double-precision floating-point arithmetic. The frequency response of the FIR-filter core was computed by getting the impulse response and taking its DFT, this is depicted with the dotted line. In this case, the MSE is $1.16 \times 10^{-4}$ Table 5 shows the synthesis report for the FIR-filter co re.

\begin{tabular}{|l|l|}
\hline \multicolumn{1}{|c|}{ Parameter } & \multicolumn{1}{c|}{ Value } \\
\hline Logic utilization & $27 \%$ \\
\hline Combinational ALUTs & $11,598 / 48,352(24 \%)$ \\
\hline Dedicated logic registers & $1,947 / 48,352(4 \%)$ \\
\hline Total block memory bits & $0 / 2,544,192(0 \%)$ \\
\hline DSP block 9-bit elements & $100 / 288(35 \%)$ \\
\hline $\begin{array}{l}\text { Maximum operating frequen- } \\
\text { cy }\end{array}$ & $103.92 \mathrm{MHz}$ \\
\hline
\end{tabular}

Table 5: Synthesis report for the FIR core with $\mathrm{N}=50$.

The FIR-filter core requires the $27 \%$ of the resources and reaches up a maximum operating frequency of $103.92 \mathrm{MHz}$.

In the case of the IIR-filter core, we designed a 12th order Butterworth band-pass filter with cutoff frequencies $0.10625 \mathrm{rad} / \mathrm{s}, 0.11875 \mathrm{rad} / \mathrm{s}, 0.1025 \mathrm{rad} / \mathrm{s}$, and $0.1225 \mathrm{rad} / \mathrm{s}$. The core was parameterized with 6 SOS sections, a word length of 16 bit, a fractional part of 13 bits, and a guard bit of 8 bits. Figure 9 shows the magnitude frequency responses for the tested IIRfilter core.

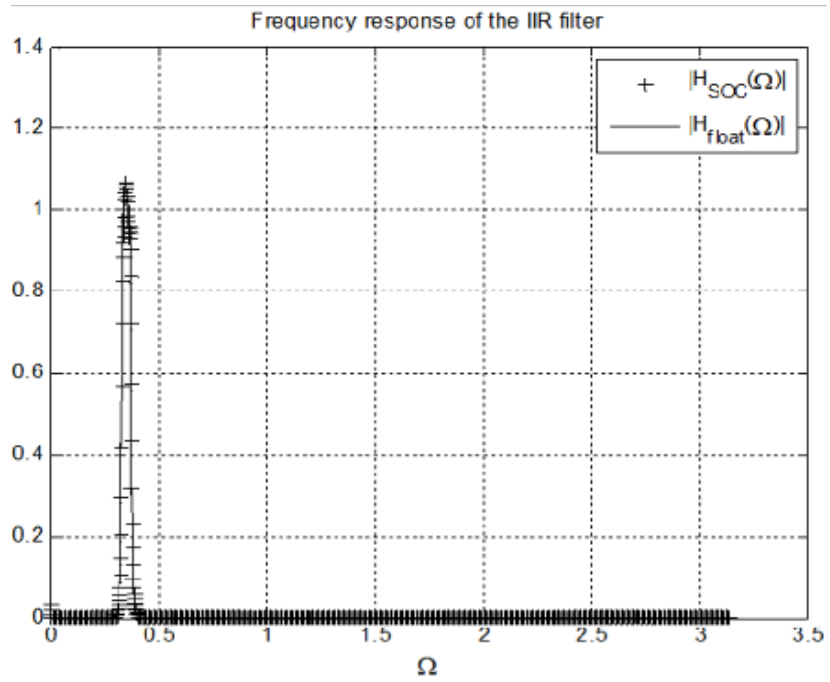

Figure 9: Frequency response of the IIR-filter core.

In Figure 9, the continuous line depicts the magnitude frequency response of the IIR filter when it is computed by simulation using double-precision floating-point arithmetic. The frequency response of the IIR-filter core was computed by getting the impulse response and taking its DFT, this is depicted with the dotted line. In this case, the MSE is $4.83 \times 10^{-5}$. 
Table 6 shows the synthesis report for the IIR-filter core.

\begin{tabular}{|l|l|}
\hline \multicolumn{1}{|c|}{ Parameter } & \multicolumn{1}{c|}{ Value } \\
\hline Logic utilization & $5 \%$ \\
\hline Combinational ALUTs & $2,201 / 48,352(5 \%)$ \\
\hline Dedicated logic registers & $504 / 48,352(1 \%)$ \\
\hline Total block memory bits & $0 / 2,544,192(0 \%)$ \\
\hline DSP block 9-bit elements & $288 / 288(100 \%)$ \\
\hline $\begin{array}{l}\text { Maximum operating } \\
\text { frequency }\end{array}$ & $85.81 \mathrm{MHz}$ \\
\hline
\end{tabular}

The IIR-filter core requires the $5 \%$ of the resources and reaches up a maximum operating frequency of 85.81 MHz.

The FFT core was parameterized with 1024 points, a word length of 16 bit, a fractional part of 15 bits, and a total gain of $2^{-4}$. Figure 10 shows the frequency responses for the tested FFT core.

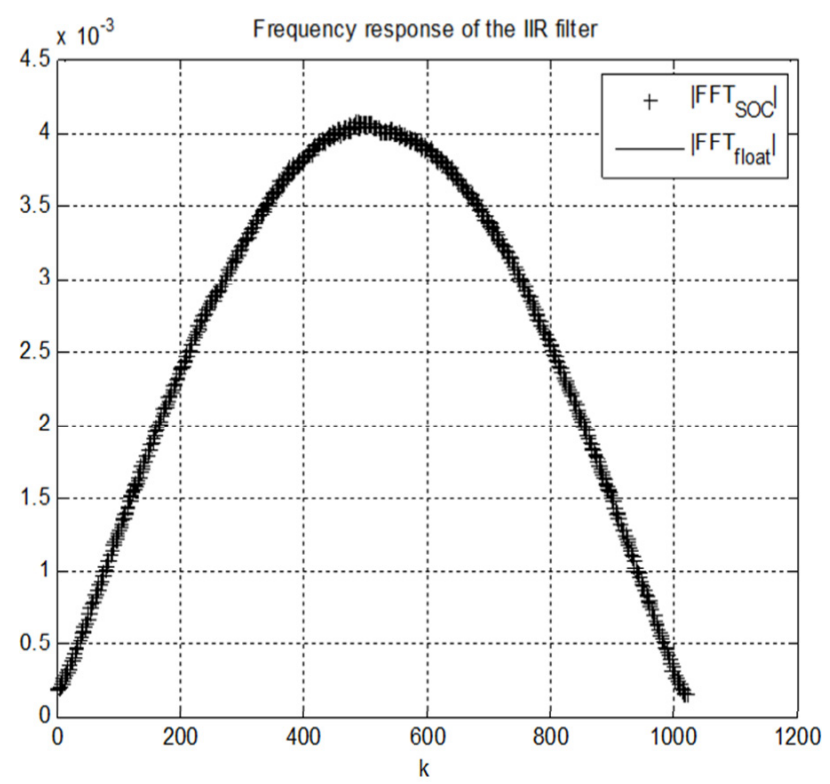

Figure 10: Frequency response of the FFT core.

In this case, we computed the FFT for the signal $x[n]=\{-0.002098083496094,0.001953125,0,0, \ldots, 0\} \vee 0 \leq n<1024$ . In Figure 10 the continuous line depicts the FFT computed by simulation using double-precision floating-point arithmetic; the dootted line depicts the FFT computed by the core. In this case, the MSE is $1.64 \times 10^{-9}$.
Open cores for digital signal processing. Valderrama et al.

Table 7 shows the synthesis report for the FFT core.

\begin{tabular}{|l|l|}
\hline \multicolumn{1}{|c|}{ Parameter } & \multicolumn{1}{c|}{ Value } \\
\hline Logic utilization & $69 \%$ \\
\hline Combinational ALUTs & $1,085 / 48,352(2 \%)$ \\
\hline Dedicated logic registers & $33,095 / 48,352(68 \%)$ \\
\hline Total block memory bits & $73,728 / 2,544,192(3 \%)$ \\
\hline DSP block 9-bit elements & $32 / 288(11 \%)$ \\
\hline $\begin{array}{l}\text { Maximum operating } \\
\text { frequency }\end{array}$ & $115.3 \mathrm{MHz}$ \\
\hline
\end{tabular}

Table 7: Synthesis report for the FFT core with $\mathrm{N}=1024$.

The FFT core requires the $69 \%$ of the resources and reaches up a maximum operating frequency of 115.3 $\mathrm{MHz}$.

\begin{tabular}{|l|l|}
\hline \multicolumn{1}{|c|}{ Parameter } & \multicolumn{1}{c|}{ Value } \\
\hline Logic utilization & $100 \%$ \\
\hline Combinational ALUTs & $33,568 / 48,352(69 \%)$ \\
\hline Dedicated logic registers & $39,428 / 48,352(82 \%)$ \\
\hline Total block memory bits & $\begin{array}{l}494,464 / 2,544,192(19 \\
\%)\end{array}$ \\
\hline DSP block 9-bit elements & $288 / 288(100 \%)$ \\
\hline $\begin{array}{l}\text { Maximum operating } \\
\text { frequency }\end{array}$ & $55.05 \mathrm{MHz}$ \\
\hline
\end{tabular}

Table 8: Synthesis report for the OpenRISC-MinSoCbased DSP SoC.

In this case, the OpenRISC-based DSP SoC requires the $100 \%$ of the resources and reaches up a maximum operating frequency of $55.05 \mathrm{MHz}$. The constraint in operating frequency is due to the MinSoC SoC and not the DSP Cores.

\section{CONCLUSION}

We designed DSP Cores FIR filter, IIR filter, FFT, which are compatible with the Wishbone bus. These cores allow the construction of DSP SoC systems based on the OpenRISC processor. These three DSP cores are parameterizable through VHDL generics and they have easy-to-use hardware/software interfaces. The three DSP cores we designed are the only of their kind in the OpenCores community because of the broad DSP functions availability, the Wishbone compatibility, the flexibility, and speed performance. The three cores have been tested on Altera FPGA devices Cyclone II and Stratix II. 


\section{ACKNOWLEDGEMENTS}

Juan Camilo Valderrama-Cuervo thanks Prof. López-Parrado for the given support and teachings. Alexander López-Parrado thanks Colciencias for the scholarship, and he also thanks Universidad del Quindío for the study commission.

\section{REFERENCES}

1. Sanjit K. Mitra. "Digital Signal Processing: A Computer-Based Approach," 4th edition, McGraw-Hill, 2010.Meyer-Baese, Uwe. (2005). Fourier Transform.

2. Uwe. Meyer-Baese, Digital Signal Processing with Field programmable Gate Array (págs. 343-391). USA: Springer.

3. Altera Corporation. FIR Compiler. 2011. http://www.altera.com/literature/ug/fircompiler_ ug.pdf

4. Xilinx Corporation. FIR Compiler. 2011. http://www.xilinx.com/products/intellectual-property/FIR_Compiler.htm

5. Altera Corporation. FFT MegaCore Function. 2011. http:/www.altera.com/literature/ug/ug fft.pdf

6. Xilinx Corporation. Fast Fourier Transform (FFT). 2011. http://www.xilinx.com/products/ intellectual-property/FFT.htm

7. OpenCores. OpenRISC 1000 Architecture Manual. 2012. http://opencores.org/ websvn, filedetails? repname $=$ openrisc \&path $=\% 2$ Fopenrisc $\% 2$ Ftrunk $\% 2 \mathrm{Fdocs} \% 2$ Fopenriscarch-1.0-rev0.pdf

8. OpenCores. Wishbone Revision B.3 Specification. 2011. http://cdn.opencores.org/downloads/wbspec_b3.pdf

9. A. López-Parrado, J. C. Valderrama-Cuervo. WDSP Project. 2013. http://opencores.org/ project,wdsp.

10. S. He and M. Torkelson. A New Approach to Pipeline FFT Processor. Proceedings of IPPS '96 the 10th International Parallel Processing Symposium. Honolulu, USA. 1996. pp. 766-770.

11. A. López-Parrado, J. Velasco-Medina, J. A. Ramírez-Gutiérrez. Efficient hardware im plementation of a full COFDM processor with robust channel equalization and reduced power consumption. Revista de la Facultad de Ingeniería de la Universidad de Antioquia. N. ${ }^{\circ} 68$ pp. 48-60. 2013.

12. OpenCores. OR1200 Project. 2012. http://opencores.org/or1k/Main_Page.

13. Raul Fajardo et. al. MINSOC Project. 2013. http://opencores.org/project,minsoc.

14. Terasic Tehcnologies. TREX-S2-TMB Motherboard for Stratix II FPGA Module Data Book. 2006. http://www.terasic.com.tw/cgi-bin/page/archive_download.pl?Language=English\&No $=189 \& \mathrm{FID}=\mathrm{d} 27 \mathrm{fe} 61 \mathrm{e} 50 \mathrm{f} 8 \mathrm{~d} 9 \mathrm{c} 5 \mathrm{c} 7 \mathrm{~d} 0278 \mathrm{~b} 78 \mathrm{c} 8 \mathrm{f} 4 \mathrm{fd}$. 